\title{
(micro)Restraint of AID
}

MicroRNAs (miRNAs) are small noncoding RNAs of $\sim 21$ nucleotides in length that regulate gene expression at the post-transcriptional level. Recent studies using loss- or gain-of-function approaches have indicated a role for the miRNA miR-155 in regulating the expression of hundreds of mRNAs and in germinal-centre reactions. However, the direct target(s) of miR-155 in B cells is still unclear. Now, two papers published in Immunity identify activation-induced cytidine deaminase (AID), a potent DNA mutator that is required for antibody diversification in B cells, as a direct target for miR-155-mediated suppression.

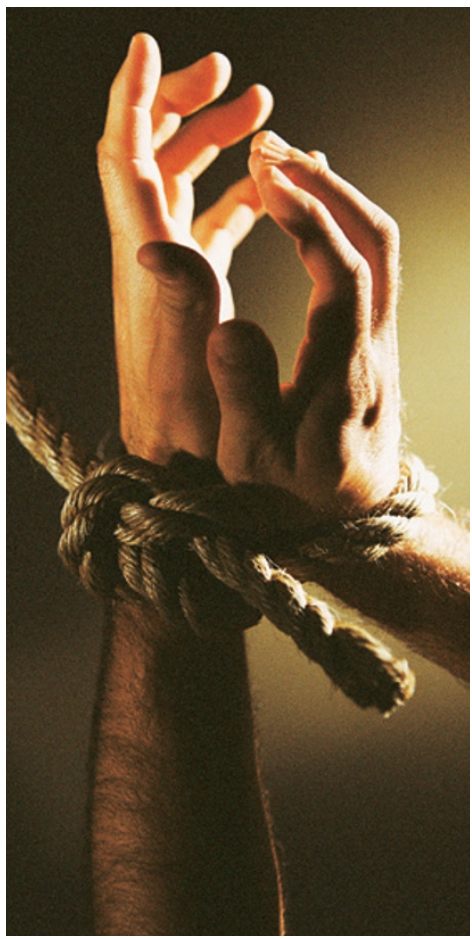

AID initiates the induction of somatic hypermutation and classswitch recombination (CSR), which together lead to the selection of highaffinity B cells in germinal centres, but because it is a DNA mutator, AID expression must be tightly regulated in the cell. Using several miRNA target-prediction algorithms, Teng et al. confirmed that Aicda, the gene encoding AID, contains a target sequence for miR-155 in its 3 -untranslated region. In addition, miR-155 expression was shown to be upregulated in B cells undergoing CSR induced by lipopolysaccharide (LPS) and interleukin-4 (IL-4).

To determine whether there is a functional interaction between miR-155 and AID, the authors generated transgenic mice expressing an Aicda transgene that contained a mutated miR-155 target site and that was tagged with green fluorescent protein (mutant Aicda mice). Following in vitro stimulation with LPS and IL-4, AID was more rapidly induced and its level of expression was higher in splenic B cells from mutant Aicda mice than in cells from control mice. In addition, increased CSR was observed in B cells from Aicda mutant mice crossed with $A i c d a^{-/-}$mice (to exclude the contribution of endogenous AID) following stimulation. So, the data show that miR-155 directly regulates the quantitative and temporal expression of AID in B cells. These observations were confirmed in vivo and further analysis revealed that the disruption of the interaction between miR-155 and Aicda in mutant mice resulted in impaired affinity maturation of B cells.
Dorsett et al. also generated mice in which the miR-155 target site in Aicda was mutated (Aicda ${ }^{155 /+}$ mice) and these mice were then crossed with $A i c d a^{-/-}$mice to produce Aicda ${ }^{155 /-}$ mice. Similar to the results of Teng et al., the amount of AID expressed and the level of CSR was higher in B cells from Aicda ${ }^{155 /-}$ mice compared with those from $\mathrm{Aicda^{+/- }}$ mice following stimulation with LPS and IL-4.

It is known that AID induces oncogenic chromosomal translocations between $M y c$ and Igh, which are associated with Burkitt's lymphoma. The authors found that the frequency of $M y c-I g h$ translocations was higher in Aicda $a^{155 /-}$ mice than Aicda ${ }^{+/-}$mice. In addition, the frequency of translocations was even higher in miR-155-deficient mice, indicating that miR-155 expression suppresses Myc-Igh translocations, at least in part, through the regulation of AID expression.

Taken together, these studies show that miR-155 suppresses the expression of AID in activated $B$ cells and that removal of this suppression results in defective affinity maturation and increased $M y c-I g h$ translocations.

Olive Leavy

ORIGINAL RESEARCH PAPERS Teng, G. et al.

MicroRNA-155 is a negative regulator of activation-induced cytidine deaminase. Immunity 28, 621-629 (2008) | Dorsett, Y. et al. MicroRNA-155 suppresses activation-induced cytidine deaminase-mediated Myc-lgh translocation. Immunity 28, 630-638 (2008) FURTHER READING Lodish, H. F. et al. Micromanagement of the immune system by microRNAs. Nature Rev. Immunol. 8, 120-130 (2008) 